\title{
On the mass determination of super-Earths orbiting active stars: the CoRoT-7 system
}

\author{
S. Ferraz-Mello ${ }^{1}$, M. Tadeu dos Santos ${ }^{1}$, C. Beaugé ${ }^{2}$, T. A. Michtchenko ${ }^{1}$, and A. Rodríguez ${ }^{1}$ \\ 1 Instituto de Astronomia, Geofísica e Ciências Atmosféricas (IAG) - Universidade de São Paulo, Rua do Matão, \\ 1226 Cep: 05508-090 São Paulo, Brazil \\ e-mail: sylvio@astro.iag.usp.br \\ 2 Observatório Astronómico de Córdoba, Universidad Nacional de Córdoba, Argentina
}

Received 3 November 2010 / Accepted 18 March 2011

\begin{abstract}
Context. Star activity makes the mass determination of CoRoT-7b and CoRoT 7c uncertain. Investigators of the CoRoT team proposed several solutions, but all but one of them are larger than the initial determinations of $4.8 \pm 0.8 M_{\text {Earth }}$ for CoRoT-7b and $8.4 \pm 0.9 M_{\text {Earth }}$ for CoRoT 7c.

Aims. This investigation uses the excellent HARPS radial velocity measurements of CoRoT-7 to redetermine the planet masses and to explore techniques for determining mass and orbital elements of planets discovered around active stars when the relative variation in the radial velocity due to the star activity cannot be considered as just noise and can exceed the variation due to the planets.

Methods. The main technique used here is a self-consistent version of the high-pass filter used by Queloz et al. (2009, A\&A, 506, 303) in the first mass determination of CoRoT-7b and CoRoT-7c. The results are compared to those given by two alternative techniques: (1) the approach proposed by Hatzes et al. (2010, A\&A, 520, A93) using only those nights in which two or three observations were done; (2) a pure Fourier analysis. In all cases, the eccentricities are taken equal to zero as indicated by the study of the tidal evolution of the system. The periods are also kept fixed at the values given by Queloz et al. Only the observations done in the time interval BJD 2454 847-873 are used because they include many nights with multiple observations; otherwise, it is not possible to separate the effects of the rotation fourth harmonic $\left(5.91 \mathrm{~d}=P_{\text {rot }} / 4\right)$ from the alias of the orbital period of CoRoT-7b $(0.853585 \mathrm{~d})$.

Results. The results of the various approaches are combined to give planet mass values $8.0 \pm 1.2 M_{\text {Earth }}$ for CoRoT-7b and $13.6 \pm 1.4 M_{\text {Earth }}$ for CoRoT 7c. An estimation of the variation of the radial velocity of the star due to its activity is also given.

Conclusions. The results obtained with three different approaches agree to give higher masses than those in previous determinations. From the existing internal structure models they indicate that CoRoT-7b is a much denser super-Earth. The bulk density is $11 \pm 3.5 \mathrm{~g} \mathrm{~cm}^{-3}$, so CoRoT-7b may be rocky with a large iron core.
\end{abstract}

Key words. planets and satellites: detection - planets and satellites: fundamental parameters

\section{Introduction}

CoRoT-7b was the first super-Earth for which mass and radius have been determined. CoRoT-7b and the recently discovered GJ 1214b (Charbonneau et al. 2009) and Kepler-10b (Batalha et al. 2011) are paradigms in the study of the physics of what exo-Earths, super-Earths, and/or mini-Neptunes can be. They set the only real constraints available to models of the formation and evolution of hot telluric planets. For this reason, it is very important to have good radius and mass determinations. The radius of CoRoT-7b, determined from the transits observed by CoRoT ${ }^{1}$, is $10100 \pm 600 \mathrm{~km}$ (Bruntt et al. 2010), a value that may be improved, but whose magnitude is nevertheless definitively established. The mass, determined from the radial velocity measurements (4.8 \pm 0.8 Earth masses cf. Queloz et al. 2009; hereafter QBM), however, does not have the same accuracy. This is because the $0.91 \pm 0.03 M_{\odot}$ (see Lanza et al. 2010), 1-2 Gyr-old G9V star CoRoT-7 (=TYCHO 4799-1733-1) is too active. The variation in the measured radial velocity comes mainly from the activity of the star whose spots determine the value of the radial velocity integrated over its disk. This activity follows roughly

\footnotetext{
1 The CoRoT space mission, launched on December 27, 2006, has been developed and is operated by CNES, with the partnership of Austria, Belgium, Brazil, ESA, Germany, and Spain.
}

the rotation period of the star (23.64 days), but in a very irregular way: the 150-day photometric observations done by CoRoT show the variation displaying full span in some times, but almost disappearing in others (see Léger et al. 2009). Because of this activity, CoRoT-7b is also the paradigm of the kind of problems that may often be found when planets with Earth-like masses are discovered. One of the aims of the investigation reported in this paper is to use CoRoT-7, for which an excellent set with 109 HARPS radial velocity measurements exists, to explore techniques that may be used when dealing with low-mass planets. We may wish that future discoveries be done around more quiet stars, but Earth-like planets are too important and we cannot discard any of them because of the central star's activity. We hope that CoRoT, KEPLER, and ground-based instruments will discover new Earths, super-Earths and mini-Neptunes that, like Kepler-10b, may be in orbit around quiet stars. However, like CoRoT-7b, they may be found around non-quiet stars, so improvement of the techniques of mass and orbit determination used to study such cases is important. This need is behind many investigations and the number of papers dealing with the mass of CoRoT-7b (QBM; Boisse et al. 2011; Hatzes et al. 2010; Pont et al. 2011) is increasing. In addition to this interest, we have to consider that HARPS, which is currently the only instrument able to make measurements of CoRoT-7 with the required 


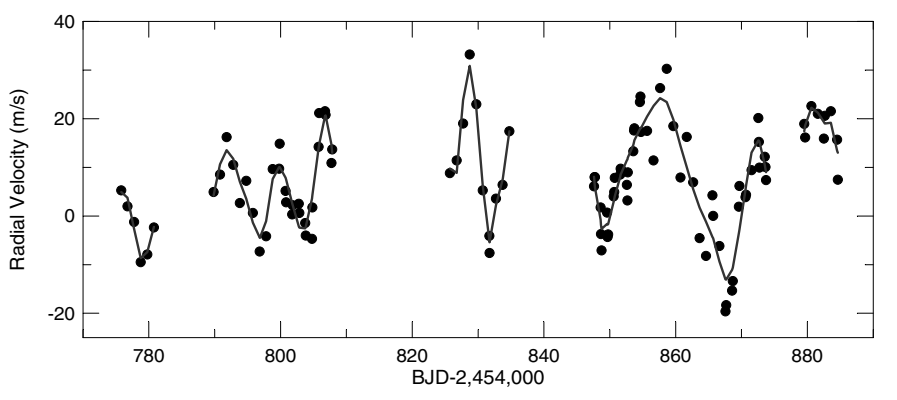

Fig. 1. Relative radial velocity measurement (dots) and the contribution of the activity as estimated with a filter using the first three harmonics of the rotational period (solid line). (Adapted from Fig. 8 of QBM.)

precision, is in demand for a great deal of other targets and we cannot foresee when a new series of measurements with the same quality of the existing one will become possible.

The nature and magnitude of the problem of the mass and orbit determination of the CoRoT-7 planets can be assessed from Fig. 1 (adapted from Fig. 8 of QBM). It shows the five sets of measurements of the radial velocity of CoRoT-7 obtained with HARPS and the estimated part of the radial velocity due to the star activity. The activity shown in Fig. 1 was obtained in QBM by means of a filter designed to eliminate periodic disturbances corresponding to the first three harmonics ${ }^{2}$ of the rotational period. These sets present patterns that are very different one from another showing that the construction of one single model for the activity in the whole interval is not possible. It also discourages the extensive use of Fourier tools (periodograms) over the whole set. We have to search for techniques able to separate the low-frequency rotation signals (periods 23.64 days and its main harmonics), from the higher frequency signal coming from the two planets (periods 0.853585 and 3.698 days cf. QBM). The difference between the periods of the two components (rotation and planets) indicates that, in this case, we may filter the data from its low-frequency parts and consider separately the highfrequency information. In the case of one continuous signal, or at least of a long discrete evenly spaced time series, the problem that we have to solve is classical and well known: we should construct and use a high-pass filter. However, the simple recipes for constructing a high-pass filter in the frequency domain cannot be used for a series with a limited amount of data, and, worse, unevenly distributed. Because of the high correlation between the absolute values of the Fourier transform (or spectral power) at different frequencies, these filters need to be constructed in the time domain.

One high-pass filter in the time domain was used in QBM in the first mass determination of CoRoT-7b and -7c. However, as discussed in Sect. 2 of this paper, the filter used then affected the high frequencies, the importance of which was downsized without apparent reason, and set the cut-off below the frequency of the main alias of the period of CoRoT-7b, thus also affecting the amplitudes corresponding to this planet (cutting out the alias, the filter also considerably downsized the signal corresponding to the actual frequency of the planet).

This paper starts with an analysis of the mass determination published in QBM's CoRoT-7c discovery paper (Sect. 2) and then proposes a self-consistent algorithm founded on the filtering

\footnotetext{
${ }^{2}$ We follow the common usage in physics where $N$ th harmonic means an oscillation whose frequency is $N$ times the fundamental frequency, so the fundamental frequency $2 \pi / P_{\text {rot }}$ is the first harmonic, the second harmonic is the component whose period is $P_{\text {rot }} / 2$, and so on.
}

technique used there (Sect. 3), which is applied to the selected set of 52 observations made on 27 consecutive nights, between BJD 2454847 and BJD 2454873 (Sect. 4) to obtain the masses of the planets. The analysis was restricted to this set of dates because in ten nights of this set, three observations were done, spanning about four hours between the first and last observations in the night. This is not enough to completely destroy the aliasing due to the almost uniform spacing between observations done on consecutive nights, but it allows us to distinguish between two solutions with forced periods equal to the transit period and its alias (see Hatzes et al. 2010, Fig. 7). It is worth stressing that, in a series made of observations taken always near the same hour in the night, no mathematical tool exists for distinguishing between one frequency and its aliases. In Sect. 4, the dependence of the results on the filter parameters is also discussed. In Sects. 5 to 7 , we present the resulting estimate of the star activity and analyze the residuals obtained by subtracting the activity from the observed radial velocities. Alternative techniques are discussed in Sects. 8 and 9. The approach discussed in section 8 follows a sugestion by Hatzes et al. (2010) and only uses the observations from nights where multiple observations were done. This is of particular importance because it allows an analysis that is independent of any explicit hypotheses on the behavior of the star activity. These observations are analyzed here with the help of a biased Monte-Carlo technique allowing confidence intervals to be obtained. The approach discussed in Sect. 9 is a classical multiperiod Fourier analysis. It differs from other approaches using a Fourier decomposition because no a priori periods are used here. The periods of the solution are those allowing us to get the best fit of the observations to a multiperiodic function. In Sect. 10, we present some simulation results taking tidal interactions into account that show the circularization of the orbits, thus justifying the adoption of zero eccentricities for both planets. At last, we proceed with the discussion of the results and the conclusions.

\section{Analysis of the first mass determination}

The analysis of the mass determination published in the CoRoT7c discovery paper (QBM) is the first step in this study, and the main point to be considered concerns the filtering properties of the procedure used there. Is it equivalent to a high-pass filter? To know that, we computed the Fourier transforms ${ }^{3}$ of the given data and of the residuals obtained in QBM after subtracting the activity, respectively, and compare them. The result presented in Fig. 2 shows that, indeed, the used procedure completely filtered the low frequencies (the transform of the filtered series is close to zero for all frequencies below $0.22 \mathrm{~d}^{-1}$ ). However, it also affected the high-frequency components. The strong alias of the period of CoRoT-7b, at 5.925d (indicated with $\mathrm{B}^{\prime}$ in Fig. 2), disappeared. The signal of CoRoT-7c was less affected by the filter, but it was downsized without apparent reason, since no low-period terms were included in the filter. The main reason for this result is that the filtering was done on the raw data, without taking the part of the signal due to the planets into account. We may guess that the incommensurability of these periods and the coherence time (window) used in the filter (20 days) may have played a role in the deep sculpting done by the filter at the

\footnotetext{
3 Spectra obtained using date-compensated discrete Fourier transforms (DCDFT; cf. Ferraz-Mello 1981). DCDFT differs from usual Lomb periodograms, because they also consider the constant component, whose neglect may affect the height of the peaks (see discussion on floatingmean periodograms in Cumming et al. 1999).
} 


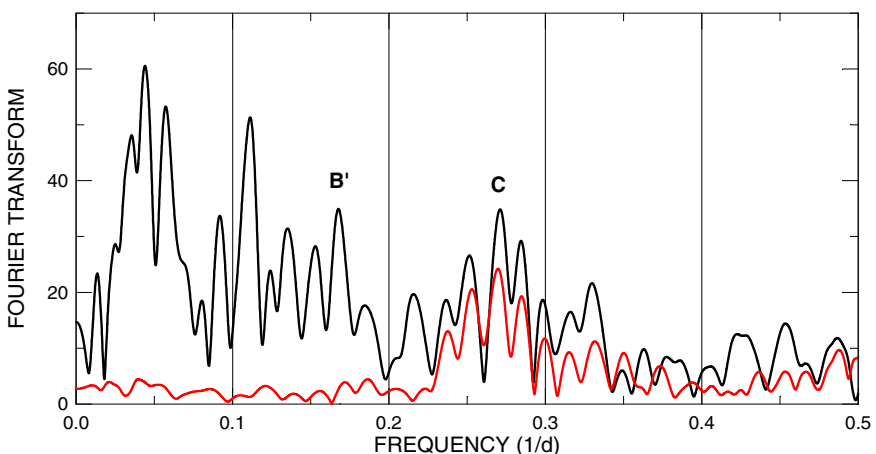

Fig. 2. Fourier transform (DCDFT) of the measured radial velocities (black) and of the values obtained in QBM after subtracting the estimated activity (red). Both transforms are presented using the same units. $\mathrm{C}$ and $\mathrm{B}$ indicate the orbital frequency of CoRoT-7c and the 5.925-day alias of the CoRoT-7b frequency, respectively.

planet frequencies and their aliases. (The average of a periodic function over a time interval not commensurable with its period is different from zero.)

A second factor may have been that fittings are more exact when there are fewer dates (as is well known). Thus, near the borders of the interval, the estimated activity will be closer to the given data than in the middle of the period. The end effect seems to be responsible for the fish-like appearance of the filtered velocities in the largest set (narrow in the extremities and wide in the middle; see the bottom panel in Fig. 8). One could think that such an appearance might result from a particular beat of the orbital frequency of CoRoT-7c and the nearby alias of the orbital frequency of CoRoT-7b, but an a posteriori plot of synthetic velocities shows that this is not so. Because of the actual phases and periods, the destructive interference of the two sine curves (at the actual discrete observation times) does not happen in the borders of the interval, but near the middle of it.

It is worth adding that one unconstrained 3-sinewave analysis of the filtered radial velocities in QBM, in the period BJD $2454847-873$, using a genetic algorithm completed with a downhill simplex, gave, as more important periods present in the filtered data, $3.495 \mathrm{~d}$ and $3.963 \mathrm{~d}$. The difference in frequency of these two periods is $0.0338 \mathrm{~d}^{-1}$, which is the inverse of $29.6 \mathrm{~d}$ (very close to the timespan of the observations used in the analysis), clearly showing the interference of the timespan of the observations in the considered subset.

\section{A self-consistent high-pass algorithm}

The high-pass filter used in QBM may be shortly described as follows. First, we define one time window (the coherence time), fixed as a guess of the number of days during which it may be reasonable to fit the given harmonic function to the activity. Then, we construct $N$ time windows of the chosen size, centered on each one of the $N$ dates of the observations and including all observations inside the window. In each window, the data are Fourier analyzed and represented by the first terms of a harmonic series whose fundamental period is the star rotation period $(23.64 \mathrm{~d})$ plus a constant. The activity at a given date is estimated as the (weighted) average of the values given to it by the harmonic representations of the signal in all subsets including the given date. For the sake of clarity, we add the following information: (i) the actual window size is fixed in such a way that the ends of the window do not separate observations done the same night (if necessary, the actual window is taken slightly

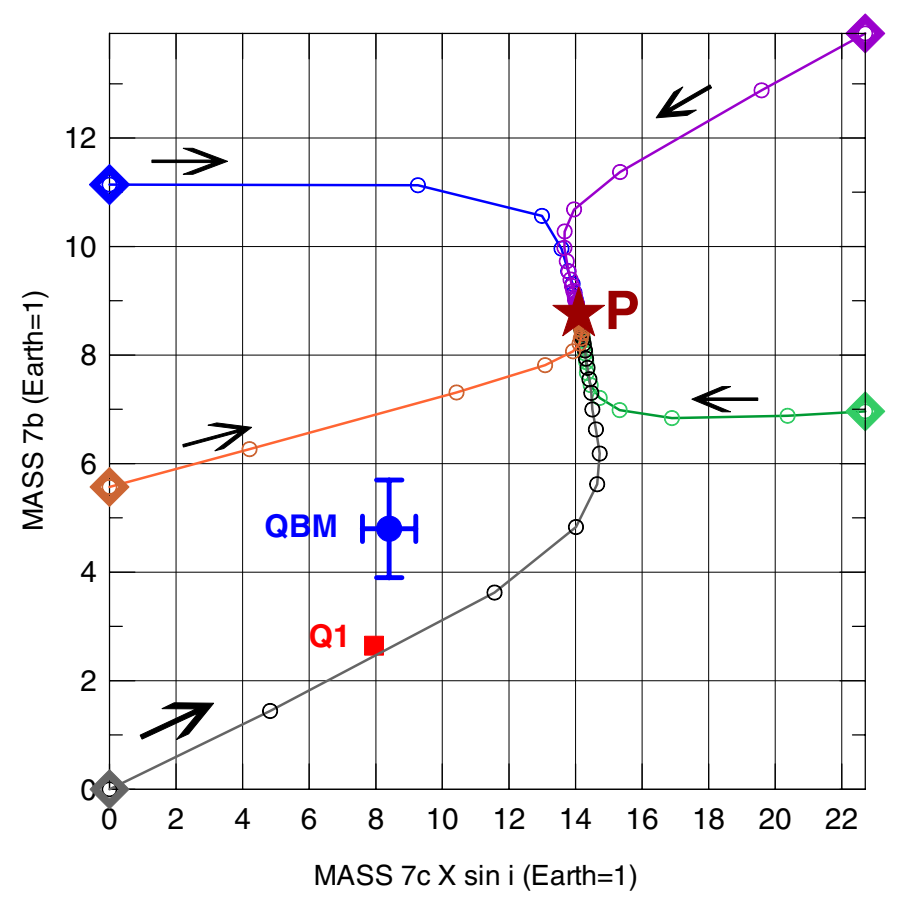

Fig. 3. Evolution of the masses in five chains of results iteratively obtained from five different sets of initial conditions (diamonds) using the 4-harmonic high-pass filter and observations done in the period BJD 2454 847-873. All of them converge to the same point $P$ (star). One of the chains started at $(0,0)$. The figure also shows the result of the first iteration in QBM (point Q1) and the mass values adopted in that paper.

larger than the nominal coherence time); (ii) near the borders, the subsets are incompletely filled as the windows extend to beyond the considered interval covering some nights where no observations were done.

In this paper, we propose an improvement to this procedure. The main change is that, now, a predicted signal corresponding to the two planets is subtracted from the radial velocities before the fitting of the harmonic function. Next, the activity is estimated as described above and subtracted from the observed radial velocities, and the resulting residuals are used to determine the masses. The new masses may then be used as first guesses in a new run of the algorithm, and this leads to an improved prediction of the signal corresponding to the two planets, and so on. The procedure is iterated as many times as needed to reach satisfactory convergence. Formally, we may say that the whole procedure defines a map $x_{n+1}=F\left(x_{n}\right)$ (here, $x$ represents the masses), which is iterated up to get $x_{n+1}=x_{n}$. One problem appearing in the actual application of this scheme is a possible slow convergence of the map. For this reason, to obtain the results discussed in this paper, we instead used an alternative accelerated map: $x_{n+1}=F\left(x_{n}+\lambda\left(x_{n}-x_{n-1}\right)\right)$ with $\lambda=1$.

In a self-consistent solution, the values of the masses used, to subtract the planets, is equal to that obtained from the filtered series. Figure 3 shows the evolution of the masses obtained in five chains of determinations using the same high-pass filter (with 4 harmonics and the same coherence time). The longest one was obtained after iterating the process from a first approximation in which the actual observed radial velocities are used without taking the planets into account (i.e. starting with the two masses equal to zero). The others started from arbitary sets of values corresponding to higher masses, represented by diamonds in Fig. 3. One may note the very slow convergence of the 
iterative procedure. The algorithm converges quickly to a line that corresponds to a linear relation between the two masses in which one of them $\left(m_{7 \mathrm{c}}\right)$ is almost constant. To have different convergence ratios along two orthogonal directions is a common feature in maps; in this case, it seems to be due to the slow separation between the planet CoRoT $7 \mathrm{~b}$ and the fourth rotation harmonic. However, notwithstanding the slow convergence, the five runs converged to the same point $P$. The reliability of the map was checked using some synthetic sets of data constructed using the first, second, and fourth rotation harmonics with the amplitudes indicated in some Fourier analyses (see Sect. 9), two sine curves of half-amplitude $6 \mathrm{~m} \mathrm{~s}^{-1}$, corresponding to two planets, and a Gaussian noise. The results are as follows: (1) when the noise is not included, the map reproduces the two planets exactly as given; (2) when noise in the range $1.7-2.5 \mathrm{~m} \mathrm{~s}^{-1}$ is added, the results for CoRoT-7b fall around the given value within $0.3 \mathrm{~m} \mathrm{~s}^{-1}$. However, the results for CoRoT-7c fall systematically $\sim 1 \mathrm{~m} \mathrm{~s}^{-1}$ below the given value. We have taken these results into account when estimating the error bars of the final results.

The analysis of the spectrographic parameters related to the star activity (FWHM, Rhk, bisector span) shows the contribution of the rotation higher harmonics to the observed activity. However, they were of little help in deciding on the kind of filtering to be used. The power spectra of these parameters in the period BJD $2454847-873$ are shown in Fig. 4. Unfortunately, the features of the spectra of the bisector span and FWHM show only small bumps at the position of the higher order harmonics, but similar bumps were found when using scrambled data, thus showing that the observed one cannot be distinguished from bumps generated by white noise so are meaningless. The corresponding data seems to be affected by the low brightness of the star. The only power spectrum showing significant peaks above the minimum level of significance is that of the index $\log R_{\mathrm{hk}}$ where peaks corresponding to higher order harmonics are clearly seen even if some offset due to the short time span of the observations used can be noted. A similar analysis using the photometric observations done in QBM showed almost no influence of the 4th harmonic and led them to neglect it in the construction of the high-pass filter. We use both filters with and without the fourth harmonic and consider them in the composition leading to the conclusions of this paper on the mass of the planets. In addition we mention that the power spectra clearly show some higher order harmonics that may affect our results. The consideration of them would require new improvements, different from those presented in this paper.

We note that the use of the rotation's fourth harmonic raises some critical questions. Indeed, its period is one fourth of $23.64 \mathrm{~d}$, that is, $5.917 \mathrm{~d}$, and one of the main aliases of the period of $7 \mathrm{~b}$ is $5.925 \mathrm{~d}$. However, aliases are defined for uniformly spaced time series with observations separated by multiples of one constant value (e.g. one sidereal day) and cannot be avoided as long as the separation between observations is kept unaltered. This is a classically known fact and it has been taken into account in the scheduling of the spectroscopic observations as soon as the observations showed this coincidence. The observations done in the period JD 2454847-873 include 52 data obtained in 27 consecutive nights, and in ten of these nights three observations were done covering about four hours. This was not sufficient to completely eliminate aliasing problems, but power spectra extended beyond the nominal Nyquist frequency showed that the perfect mirroring of the power spectrum had been avoided (see Fig. 10 of QBM). Several other tests were done. Least squares determinations simultaneously involving the

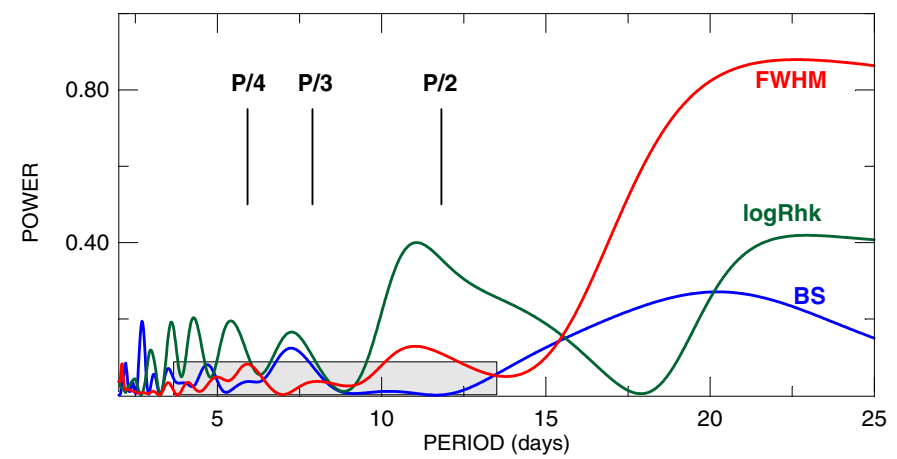

Fig. 4. Power spectra of the spectrographic parameters associated with the star activity: bisector span, FWHM and $\log R_{\mathrm{hk}}$, in the period BJD $2454847-873$. The exact location of the main rotation harmonics is indicated. The gray rectangle is below the minimum significance level. Peaks inside this rectangle can be produced by random data.

photometric period $0.853585 \mathrm{~d}$ and the fourth rotation harmonic led to correlation values in the range $0.70-0.88$ depending on the design of the experiment done and the observations used. Some of these results are worrisome, but the final test is provided by the coincidence of the convergence points in chains starting at very different mass values (like the test ones shown in Fig. 3). One characteristic of least-squares procedures involving highly correlated parameters is that the results become erratic. This has never been the case here. However, it is clear that, without the multiple-data per night policy adopted in the last periods of observations, it would be impossible to separate the rotation fourth harmonic from the alias of the period of CoRoT-7b.

In what concerns the higher harmonics, we mention that the proximity of the period of the 6 th harmonic (3.94 days) to the period of CoRoT-7c (3.698 days) is a problem with a difficult solution. They are far enough apart to be separated one from another, but the beat period of the two components (about 60 days) is much longer than the timespan of the subset (27 days). When the algorithm used in this paper is extended to include these harmonics, its convergence becomes excessively slow. The Fourier analysis of the residuals obtained with the 4-harmonic high-pass filter and an extended time interval (BJD $2454847-884$ ) indicated that the amplitude of the radial velocities due to CoRoT-7c may be affected in up to $0.5 \mathrm{~m} \mathrm{~s}^{-1}$. This possible offset will be taken into account in the final results.

\section{The mass of the planets}

The technique described in the previous sections is certainly the best one we can devise to eliminate the contributions of irregular, long-period terms from a given series of unevenly spaced observations and to get a remaining part that may be used to determine the parameters of a short-period signal (the planets). It is now applied to the observations. However, for the reasons discussed above, we abandon using all observations, but instead concentrate on the set of observations done in the period BJD 2454847-873. In addition to the aliasing problem, the consideration of the whole set - formed by five different subsets spanning 106 days plus three isolated observations one year before - is made difficult by the irregular variation in the stellar activity from the epoch of one set to the next.

Another important setting in this determination is that we concentrate on the masses and fix the periods in the values previously determined (Léger et al. 2009, and QBM). One of the reasons is that by restricting the interval under study to a set of 
only 27 days, there is no possibility of improving a period determination resulting from observations taken from a time interval four times longer. The analysis of the covariance matrix with the 27-day data shows very high correlation (0.97) between periods and phases for both planets, which means that this short set cannot be used to determine periods and phases simultaneously.

The results of this algorithm depend on the model used in the high-pass filter. Two main parameters were investigated. One of them is the model used in the interpolation to determine the activity at a given date: here, we considered both the 3 - and the 4-rotation harmonics models. In a lesser extent, the 6-harmonics models has also been considered, but the beat of the periods of CoRoT-7c and that of the 6th rotation harmonic impairs the procedure convergence. The other parameter is the coherence time, which sets the size of the window. We investigated several of them starting with the 20-day interval as used by QMB, but also considered some other values in the range 8-22 days. The results are shown in Fig. 5. As long as the coherence time is kept in a limited interval, the results do not show large variations. Also, since the codes themselves depend on some operational parameters that might affect the results, the procedures of filtering and mass determination were done with two very different codes: one, a lengthy steepest descent (diamonds) and the other a two-part code using a genetic algorithm completed with a downhill simplex (crosses). Labels indicate the corresponding coherence times. For the set shown by crosses, only the highest label (22) was shown to avoid excessive overlap of symbols and labels; the solutions with coherence times between 8 and 12 days cluster around $m_{B}=8 \pm 1.2 m_{\text {Earth }}$ and $m_{\mathrm{C}}=13.6 \pm$ $1.4 m_{\text {Earth }}$. The green rectangle indicates a joint interval of confidence. The individual statistical errors of the self-consistent determinations were estimated as $0.5 \mathrm{~m} \mathrm{~s}^{-1}$, that is, 0.7 Earth mass for $7 \mathrm{~b}$ and 1.1 for $7 \mathrm{c}$.

The sets of masses obtained for both planets show significant dependence on the used high-pass filter. The mass of CoRoT-7b shows some variations whether the fourth rotation harmonic is included or not in the model. When the fourth harmonic is included, we obtain for CoRoT-7b a mass above 8 Earth masses, while the results with only three harmonics is smaller than 8 Earth masses. In its turn, the models with three and four harmonics indicate, for CoRoT-7c, a mass around 14 Earth masses, but the results becomes determined less well when higher harmonics are included, in a way leading us to believe that the actual mass of CoRoT-7c is lower than the obtained value. The results of some runs using more observations (the 72 observations done in the interval BJD 2454845-873) also result for CoRoT-7c a mass lower than 13 Earth masses. The results also depend on the period adopted for the planets. Because of its very short period, CoRoT-7b shows more sensitivity. Fortunately, the period of CoRoT-7b is very well known from the CoRoT photometry and this source of error can be discarded.

These experiments have also shown that the results are sensitive to the adopted weighting rules. All results in this paper used, at the beginning, the standard errors published by the observers (Léger et al. 2009), which were propagated following the classical rules of the least-squares formulas for unequally weighted observations (see Linnik 1963) ${ }^{4}$. In the sequence, variances were obtained for each subset used in the filtering and were used to

\footnotetext{
4 The used classical weighting rules are able to take into account that the observations done at the dates 2454860.75 and 2454864.63 have worse quality than the others; the weight associated to them was nearly ten times less than the weight given to the more precise observations in the set.
}

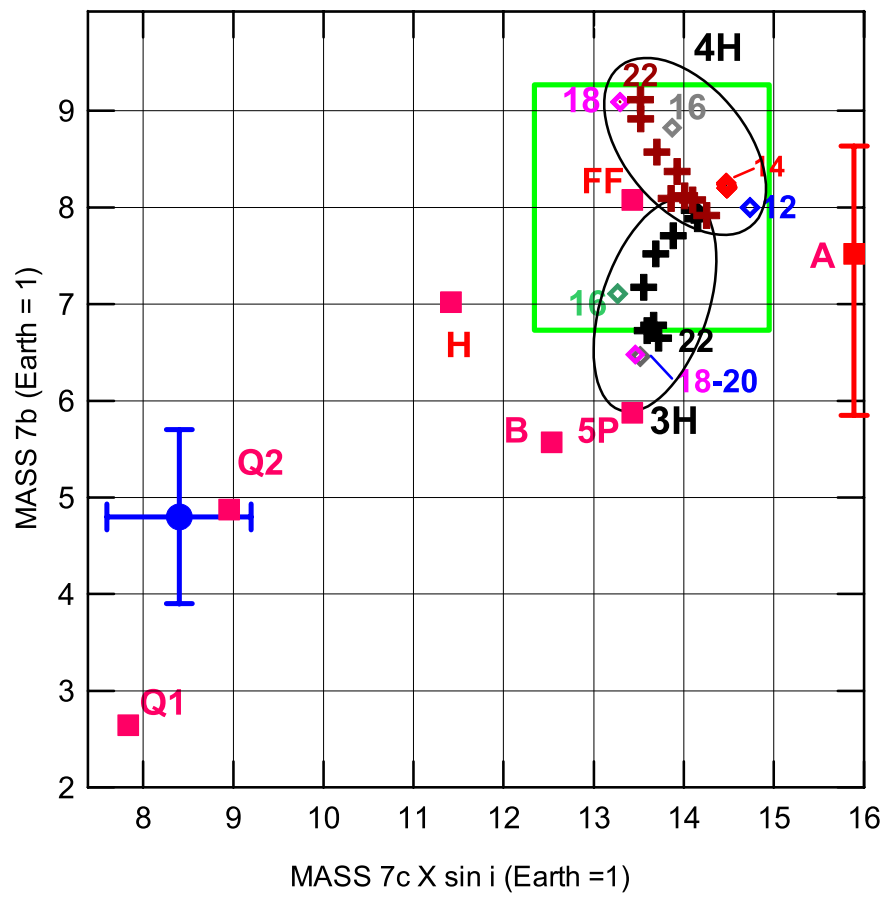

Fig. 5. Planet masses resulting from several runs of the iterative highpass filtering with 3-, 4-harmonic filters (sets $3 \mathrm{H}, 4 \mathrm{H}$ ). The other labels indicate the coherence time in days (see details in the text). The green square represents the interval of confidence of the solution given in Table 2. The large blue cross indicates the adopted solution in QBM and the red squares Q1 and Q2 the solutions corresponding to the published values of $K_{1}, K_{2}$, in QBM. The solutions A (alternative; only CoRoT 7b), B (Boisse), H (Hatzes), 5P (Fourier with 3 harmonics), FF (free Fourier) are discussed in Sects. 8 and 9.

weight the averages on each date giving the estimate of the activity. Finally, the mass determination from the filtered radial velocities cannot use the same weights as the given observations. The filtered RVs are differences between the observed RV and the estimated activity and are, therefore, affected by the errors in both these quantities. The classical formulas after which the variance $\left(\sigma^{2}\right)$ of the difference is the sum of the variances in the two quantities entering into the subtraction is used and the new weights are defined as the inverse of the resulting variances.

\section{The star activity}

Figure 6 shows the activity determined using the 3-, 4-harmonic high-pass filters (bottom) and the difference between them (top). The activities determined with both filters and various values of the coherence time are shown. For a given model, they do not show visible differences, notwithstanding the fact that these differences exist and affect the mass determination. The analysis of this figure may be summarized in a few words: (1) the filtering is very robust with respect to the chosen coherence time and model; and (2) the results are smoother when less harmonics are included in the filter.

The activity estimated here may be compared to the one estimated by Pont et al. (2011) from the analysis of the bisector span measurements. It is remarkable that the features of the activity given in Fig. 2 of Pont et al. are very similar to those shown in Fig. 6 corresponding to the 4-harmonic filtering; however, one may note that the total span of the RV due to the star activity is, there, about half of what is shown in Fig. 6. 


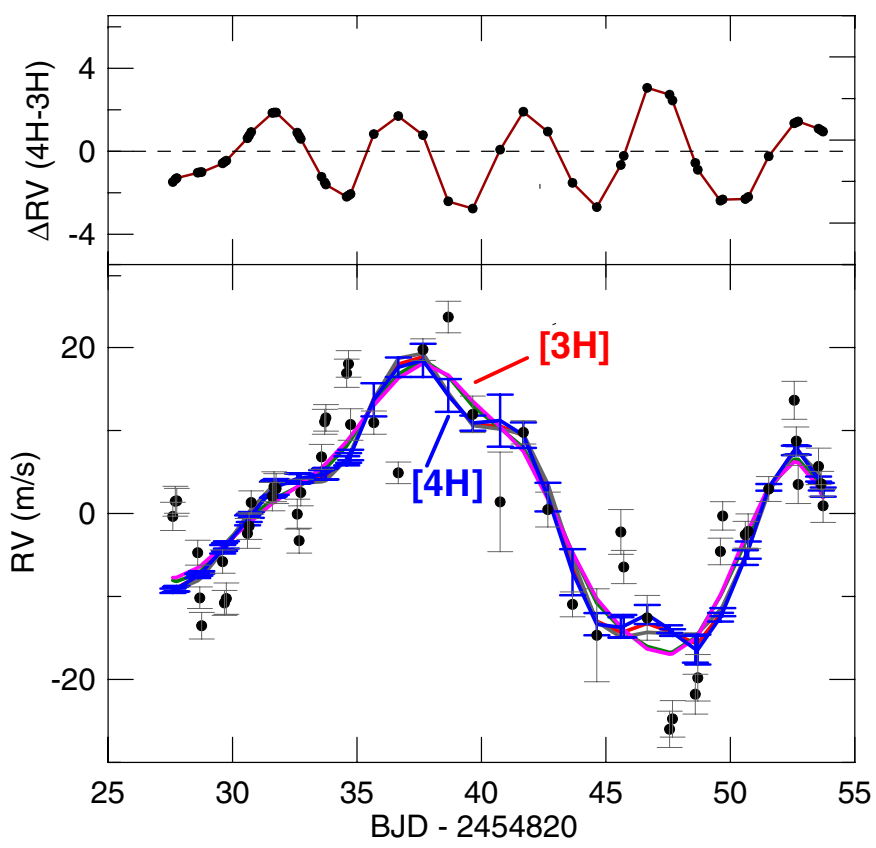

Fig. 6. Bottom: star activity resulting from the high-pass filter. The labels $[4 \mathrm{H}]$ and $[3 \mathrm{H}]$ indicate the results obtained respectively with 4 and 3 harmonics. The color coding of the components of these curves, when visible, is the same as in Fig. 7. The dots indicate the measured radial velocities with their error bars. The error bars of the activity estimated with the 4-harmonic high-pass filter are also shown. Top: difference between the results labeled $4 \mathrm{H}$ and $3 \mathrm{H}$.

\section{Quality of the new high-pass filter}

As for the QBM determination, we may compute the Fourier transforms of the residuals $V_{\text {obs }}-V_{\text {activ }}$ and compare them to that of the observations. Some Fourier transforms (DCDFT) are shown in Fig. 7 (top) for the two models and several values of the coherence time. To avoid the complication arising from many almost overlapping curves, we only present those transforms for which the height of the peak near the frequency labeled B' (alias of the orbital frequency of CoRoT-7b) nearly matches the peak of the transform of the observed data. It is worth stressing that the frequency $\mathrm{B}^{\prime}$ is not separated from the side lobes of the rotation period (which are broad because of the short time span of the used observation set). Because of this superposition, it is difficult to assess the quality of the filtering by inspection of the filtered spectra at this frequency. The composition of two frequencies in a spectrum is not just an addition since each of them carries one phase and the effect of the superposition cannot be assessed only by comparing their moduli. In this case, the superposition is reinforced by the short time span of the considered data. With a longer timespan, peaks would be sharper (as in Fig. 2) and could be separated, but using all available data in the analysis would mean to work with a discontinuous set of observations, which introduces additional (and in some extent unsolvable) difficulties in the estimation of the activity. One noteworthy effect of the superposition is the apparent enhancement of the peaks at $\mathrm{B}$ and $\mathrm{B}^{\prime}$.

The frequency of CoRoT-7c, however, is less affected, at least as long as higher order harmonics are not included.

For the sake of giving additional information on the aliasing effects, we present in Fig. 7 (bottom) an extension of the transforms to an interval of frequencies including the actual orbital frequency of CoRoT-7b (labeled B) and one alias of the orbital frequency of CoRoT-7c (labeled $\mathrm{C}^{\prime}$ ). As expected,

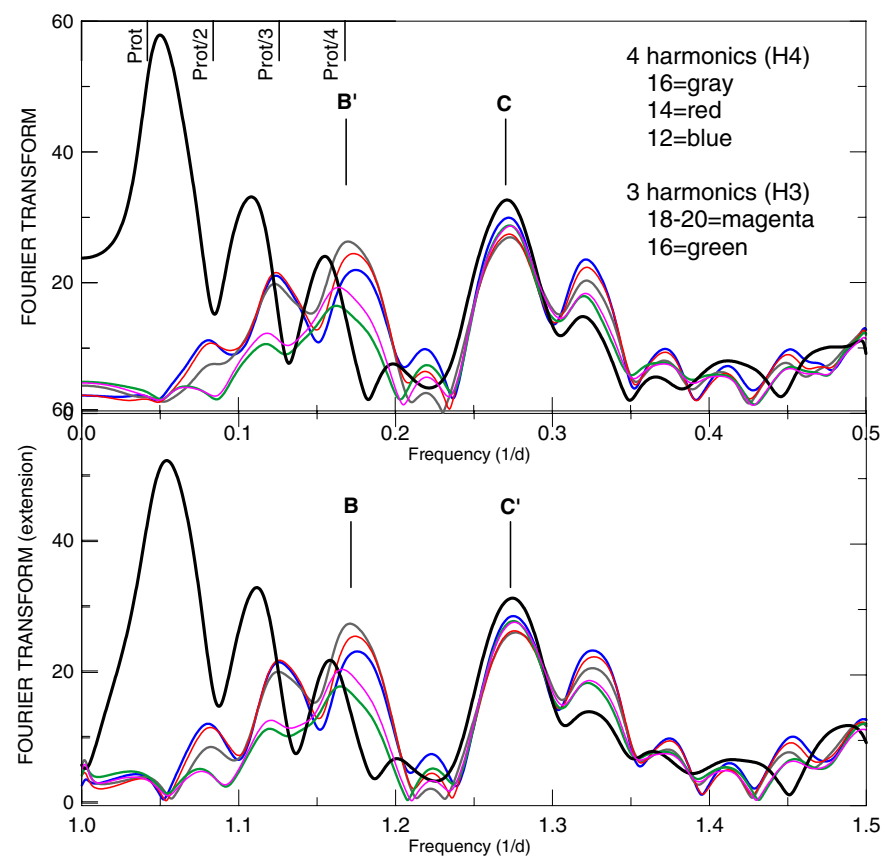

Fig. 7. Top: fourier Transforms (DCDFT) of the observed (black) and filtered data with the 3- and 4-harmonic high-pass filters in the period BJD $2454847-873$. Bottom: extension of the transforms to beyond the Nyquist frequency to show their behavior below 1 day. $B, C$ are the orbital frequencies of CoRoT $7 \mathrm{~b}$ and $7 \mathrm{c} ; B^{\prime}, C^{\prime}$ are aliases of $B, C$. The rotation period and its first harmonics are indicated on the top axis.

the transforms in the given intervals are almost identical in both plots, but not perfectly identical because of the high proportion of nights with multiple observations in the selected time interval.

The comparison of the Fourier transforms shows that the best-quality filtering was obtained with coherence times of 12-14 days when using the 4-harmonic filter and 18-20 days when using the 3-harmonic filter (the curves for 18 and 20 days are almost identical).

\section{The filtered radial velocities}

Figure 8 (Top) shows the radial velocities obtained subtracting from the measured radial velocities the activity resulting from the use of the 4-harmonic filter and coherence time $\tau=14 \mathrm{~d}$. They may be compared to the ones used in the discovery paper (Fig. 8 Bottom). The main differences appear in the beginning of the interval, where the filtered radial velocities appear much higher in our results than in QBM and in the middle of the interval where the contrary occurs. In the date 42 , in our results, a destructive beat occurs between the RV sine curves corresponding to the two planets, at the actual observing times. We should stress that the epoch BJD 2454820 used in the plots and calculations of this paper is not the same epoch used in other papers. It is an arbitrary epoch close to the actual dates and allows the phases (i.e. the longitudes at the fixed epoch) to depend less strongly on the periods, thus making the numerical procedures more robust. In addition, in all steps one additive constant representing the radial velocity of the planetary system is determined together with the other unknowns.

\section{A model-independent approach}

The use of a high-pass filter allowed us to separate parts of the RV measurements due to high- and low-frequency components. 


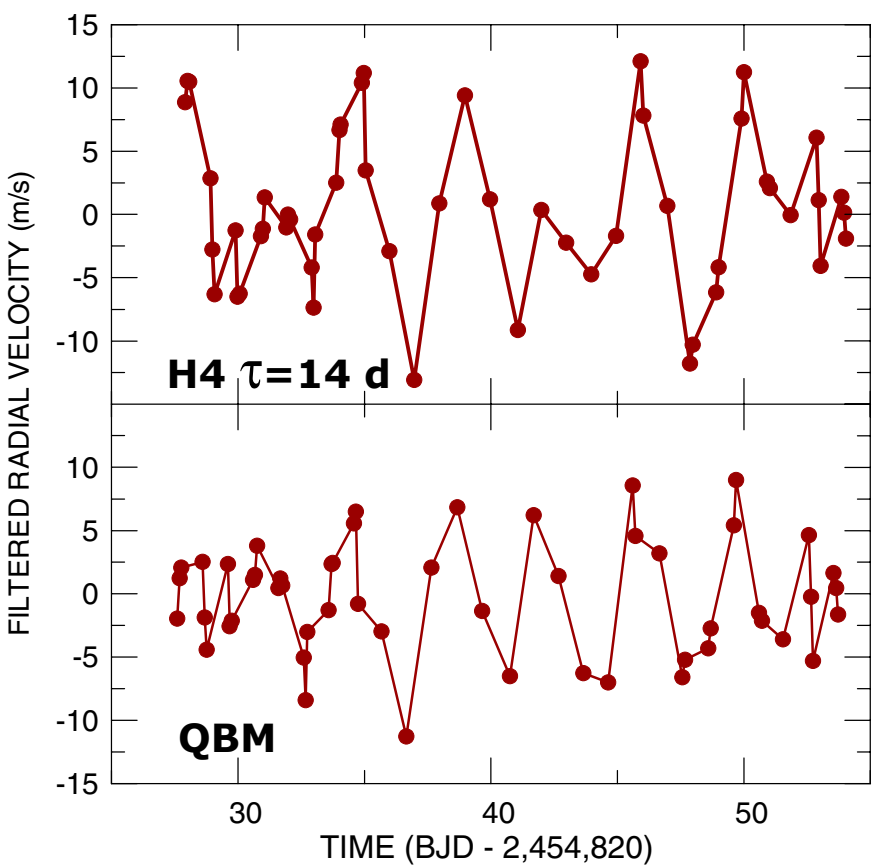

Fig. 8. Filtered radial velocities obtained with the 4-harmonic highpass filter and coherence time $\tau=14 \mathrm{~d}$ (top) compared to those given in QBM (bottom).

The results are, however, model-dependent. The high-pass filtering has given statistically coherent estimates for the mass of CoRoT-7c, but that result can carry some systematic effects of some higher order rotation harmonics. For CoRoT-7b, the filtering led to two different solutions. From the purely statistical point of view, the 4-harmonic filtering should be better than the 3-harmonic filtering. However, because of the aliasing involving the rotation fourth harmonic and the orbital period of CoRoT-7b, it is convenient to confirm the results with some alternative technique independent of assumptions involving the rotation period and its harmonics. An alternative approach suggested by Hatzes et al. (2010, and paper in prep.) in which only data from nights in which multiple observations were done are used, and which assumes that in the four hour time span of one observation night, the activity of the star does not change and may be fixed as the same for the three observations ${ }^{5}$. There are ten nights in the interval 2454847-873, in which three consecutive observations were done. Then, the problem is to fit those observations with two planetary sine waves (the eccentricities may be assumed equal to zero as discussed in Sect. 10) and a set of ten independent constants added to the data in the corresponding dates. The main problem with this approach is, however, the small number of degrees of freedom in the best-fit problem. We have 30 data and 14 unknowns. The number of degrees of freedom is 16 . In analogy with a chi-square distribution, we may guess that every solution leading to a w.r.m.s. $\leq 0.21 \mathrm{~m} \mathrm{~s}^{-1}$ higher than the minimum must be considered as belonging to the standard interval of confidence (see Press et al. 1986). To improve the determination, we have chosen to add to these observations those from 16 other nights with two observations. We have then 62 data and 30 unknowns. The number of degrees of freedom becomes 32. This is twice what we would have if only using

\footnotetext{
5 The results from the used RV modeling indicate that variations are smaller than the observational errors. However, in one extreme (and infrequent) case, the estimated activity varied by $0.8 \mathrm{~m} \mathrm{~s}^{-1}$ between the first and the last observation of the night.
}

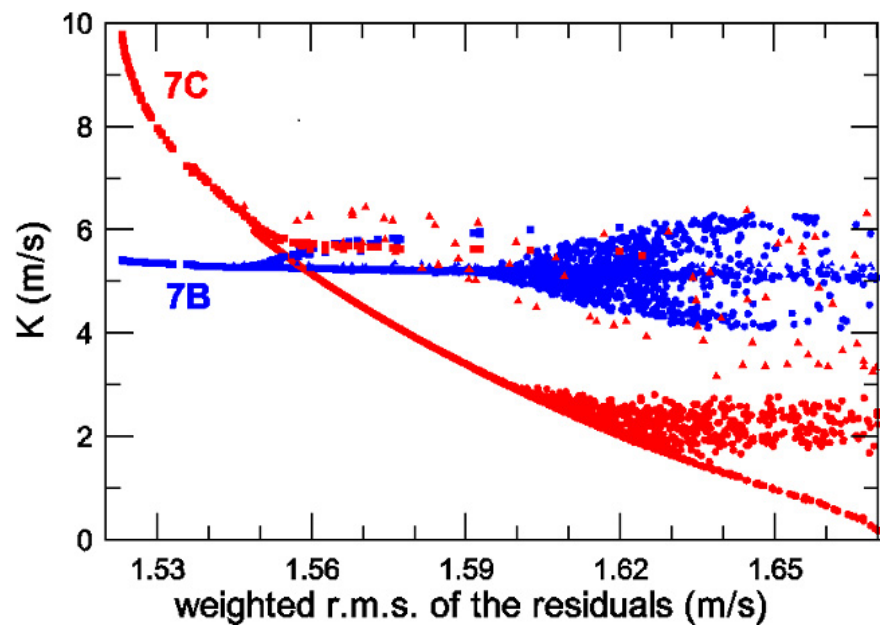

Fig. 9. Good fits obtained with a biased Monte-Carlo under the condition w.r.m.s. $\leq 1.67 \mathrm{~m} \mathrm{~s}^{-1}$. The blue dots correspond to CoRoT-7b and the red ones to CoRoT-7c.

the ten nights with three observations each, and it means a less broad confidence interval, including only the solutions leading to a w.r.m.s. $\leq 0.16 \mathrm{~m} \mathrm{~s}^{-1}$ larger than the minimum.

To assess the set of all these "good-fit" solutions, we may use the same biased Monte-Carlo technique as used to obtain good fits for the planets HD 82943 b,c (Ferraz-Mello et al. 2005). As before, the frequencies were fixed at the values given in QBM (they are well determined) and only four unknown planetary parameters were considered: the two masses and the two phases. The (biased) random search produced thousands of solutions. Those with w.r.m.s. $\leq 1.67 \mathrm{~m} \mathrm{~s}^{-1}$ are shown in Fig. 9, where we superpose the results for the half-amplitudes $\mathrm{K}$ of the two planets. The mass of CoRoT-7b is constrained to the interval $m_{B}=7.2 \pm 1.4 m_{\text {Earth }}$ (i.e. $4.1 \leq K \leq 6.2 \mathrm{~m} \mathrm{~s}^{-1}$ ) and its longitude at the epoch BJD 2454820.0 is $200 \pm 7 \mathrm{deg}$. The mass of CoRoT-7c, however, is not constrained by this set of observations. Fits with w.r.m.s. $\leq 1.67 \mathrm{~m} \mathrm{~s}^{-1}$ exist for masses in a broad interval: $m_{\mathrm{C}} \sin i \leq 22 m_{\text {Earth }}\left(K_{\mathrm{C}} \leq 9.8 \mathrm{~m} \mathrm{~s}^{-1}\right)$.

The poor constraining of the solution for CoRoT-7c deserves some discussion. The alternative technique proposed by Hatzes et al. (2010) is good for separating low- and high-frequency contributions. The period of CoRoT-7c is not short enough to be considered as low. In some nights, the variations in the RV due to CoRoT-7c may reach $2 \mathrm{~m} \mathrm{~s}^{-1}$ in five hours. It is generally less, and not distinguishable from contributions coming from low-amplitude, long-period terms. We cannot avoid the random process from mimicking, in the activity, contributions with the same period as CoRoT-7c and from combining them with the planet RV. The results are not accurate, but they are important because of their independence with respect to assumptions on the activity behavior.

The best-constrained fit, defined as the leftmost point in Fig. 9, corresponds to the mass $m_{B}=7.5$ Earth masses. This fit and the interval of confidence corresponding to the maximum span of the solutions in the considered interval is shown by point A in Fig. 5. It is important to stress that each set of masses and phases of the two planets obtained in this experiment corresponds to one solution whose residuals fit the observations better than $1.67 \mathrm{~m} \mathrm{~s}^{-1}$ (w.r.m.s.) and cannot be discarded. The density of the points in Fig. 9 has no meaning (but instead reflects the strategy used to construct the sets). Only the error bar for $m_{B}$ is given. It was not possible to get a good determination for $m_{\mathrm{c}} \sin i$ 
and the corresponding error bar would be larger than the width of Fig. 5.

\section{Fourier approaches}

As a first guess, one could assume that the observations can be represented by the sum of several periodic functions and use conventional non-windowed Fourier analyses to determine their parameters (amplitudes, periods, phases). The solutions indicated by $\mathrm{B}$ and $\mathrm{H}$ in Fig. 5 are those obtained by Boisse et al. (2011) and Hatzes et al. (2010) from the Fourier analysis of the observations. The Boisse et al. solution is the best fit of five sine curves to the radial velocities, three of which have the periods of the rotation and its first harmonics. For that solution, besides the 52 points used in this paper, the remaining nine points of the latest observational subset were also considered. The Hatzes et al. solution was obtained using all observations and a pre-whitening plus filtering procedure based on periodograms; nine different sine curves contributed to the construction of the solution.

If we proceed in the same way as Boisse et al. (2011), but consider only the set of 52 observations used in previous sections, the result is shown by the symbol 5P in Fig. 5. In fact, we did a great deal of Fourier analyses of the measurements, which remained unpublished only because they assume a periodic behavior in the activity, which is very improbable. Every function in a finite interval may be represented by a Fourier series, but in the present case, the main period needs to be the rotation period. Such a periodic structure is not seen in the residuals published by QBM (shown in Fig. 1 above), in our own activity curve (shown in Fig. 6 above), in the activity as determined by Pont et al. (2011) (their Fig. 1) or in the photometric series produced by CoRoT (Léger et al. 2009). In all these plots there is some kind of repetition associated with the rotation period, but, in some sections, the curve appears dominated by the rotation period, while in others by the rotation harmonics, and so on. None of the partial curves appearing in Fig. 1 shows the 23.64 period indicated by photometry. Irregular behavior is also seen in the light curve, whose periodogram clearly shows the rotation period and its harmonics, but in some sections of it no variations are seen, while in others the rotation period is well marked.

We report here only one of the experiments that consisted of several steps: (a) The periods were initialized in relatively broad intervals bracketing the rotation period and its harmonics, allowing for possible differences due to either the physics of the activity or the beat between the actual periodic signal and the sampling set (actual observation dates); (b) The approximated, more probable elements (including the periods) were determined via a chain of best fits to the data; (c) in each step, the elements were determined by the simultaneous best fit of $N$ trigonometric components. The process was stopped when the addition of one new term was no longer able to significantly improve the results (F-test), and the scrambled data produced spectra with peaks of the same size as the ones obtained with the real data. In such a case, including more unknowns in the process may lead to high correlation between the unknowns and to results that may become indistinguishable from artifacts.

The eventual results of the free Fourier approach are shown in Fig. 5 (point FF) and detailed in Table 1. The error bars were estimated with a biased Monte Carlo sampling of the neighborhood of the solution, which showed very asymmetric distributions in some cases.

It is important to stress that the weakest of the five terms is already very uncertain as both the spectrum of the residuals in the previous step, and the a posteriori F-test have shown that no
Table 1. Fourier decomposition of the data in the time interval $2454847-873$. The resulting w.r.m.s. of the residuals is $1.76 \mathrm{~m} \mathrm{~s}^{-1}$.

\begin{tabular}{ccc}
\hline \hline Period (d) & $K\left(\mathrm{~m} \mathrm{~s}^{-1}\right)$ & $\begin{array}{c}\text { Mass (Earth }=1) \\
\times \sin i\end{array}$ \\
\hline $21.16 \pm 0.6$ & $15.0_{-1.4}^{+0.6}$ & \\
$3.70 \pm 0.04$ & $6.0 \pm 0.9$ & $13.4 \pm 2.0$ \\
$0.850 \pm 0.002$ & $5.8 \pm 1.2$ & $8.1 \pm 1.5$ \\
$11.7_{-0.7}^{+0.3}$ & $4.0 \pm 1.0$ & \\
$5.09 \pm 0.15$ & $2.4_{-0.9}^{+1.4}$ & \\
\hline
\end{tabular}

actual improvement was obtained by the addition of this term to the solution. One important by-product of this analysis concerns the conjecture of the existence of one third planet at $P=9.2 \mathrm{~d}$, which cannot be studied with the other techniques described in this paper, since they do not allow such a slow periodic variation to be distinguished from the star activity. Peaks corresponding to periods around nine days recur in all analyses, since the beginning of this investigation. They can be seen, for instance, in the spectra of the raw data in Figs. 2 and 7. However, always, when a monochromatic filter is used and a sinus curve with the amplitude and period of the rotation is subtracted, that peak disappears. We conjecture that it results from a complex beat between the rotation period, the sampling dates, and the planets themselves.

\section{The eccentricities}

In the mass/orbit determination presented in this paper, the eccentricities were taken equal to zero. Indeed, an analysis of the dynamical problem shows that the tidal dissipation in the planet CoRoT-7b and its gravitational interaction with CoRoT-7c damp both eccentricities. Simulations were done where the forces due to the tide raised in the planet (cf. Mignard 1979) were added to the gravitational ones. In all of them, the osculating eccentricities of the two planets stabilize in values of about $10^{-5}$ and $10^{-4}$ in a few tens of Myr, whichever initial eccentricities and inclinations are considered. In the solution shown in Fig. 10, the planets are initially on circular orbits with semi-major axes 0.0175 and $0.0456 \mathrm{AU}$ (like CoRoT-7c) in two planes with a mutual inclination of 40 degrees. The chosen initial eccentricities do not affect the solution because, immediately after the beginning of the simulation, the gravitational interaction of the two planets forces the eccentricities to be higher than 0.1 and 0.01 respectively. (In the coplanar simulations they jump to the equilibrium values fixed by the mutual perturbations; cf. Mardling 2007; Rodríguez 2010; Rodríguez et al. 2011.) Thereafter, the tidal dissipation in the inner planet starts dissipating the energy of the system, making the orbit of CoRoT-7b slowly spiral down towards the planet and become circular while the semi-major axis of CoRoT-7c remains almost unchanged. In addition, the exchange of angular momentum between the two planets also damps the eccentricity of CoRoT-7c and drives the planets to an equilibrium configuration (see Mardling 2011). It is worth recalling that after the circularization of the orbit of CoRoT-7b, the tidal friction in this planet almost ceases (see Ferraz-Mello et al. 2008) and no longer continues to significantly affect the evolution of the system. In some coplanar runs starting with eccentric orbits, the final eccentricities are even lower than the ones shown in Fig. 10. 
S. Ferraz-Mello et al.: Planetary mass determination of super-Earths orbiting active stars

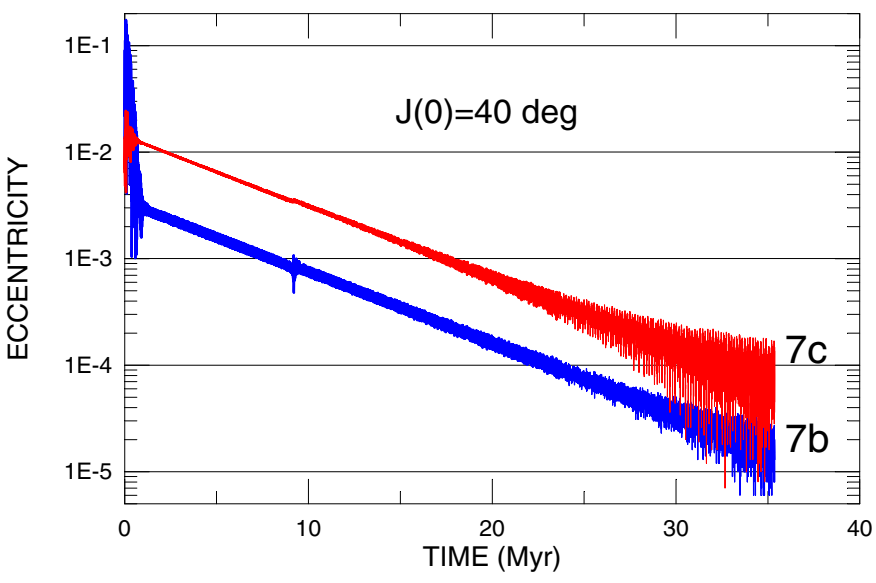

Fig. 10. Orbit evolution of two planets with masses 8 and 15 Earth masses in a CoRoT-7-like system with mutually inclined orbits. The damping of the eccentricities is due to the tides on the inner planet only.

\section{Discussion}

Three independent approaches were used to assess the masses of the exoplanets CoRoT-7b and CoRoT-7c, notwithstanding the difficulties created by the activity of the star that contaminates the measured velocities not as a jitter, but as a dominant signal some two to three times more intense than the planetary contributions. Before comparing the results, it is worth stressing that the residuals of the three analyzed models have weighted rms less than $1.9 \mathrm{~m} \mathrm{~s}^{-1}$, which may be considered good given the irregular activity of the star. However, we have to prevent against using the minimum w.r.m.s. of the models to mutually compare them, because, by definition, these quantities are minima of the residuals with respect to a given model, so may be considered as model-dependent. They are only used to make comparisons within a given model.

The introduction of a self-consistent algorithm improved the filtering used in QBM and resulted in considerably higher masses than the ones obtained previously. Influencing the results is the adoption of a 4-harmonic filter in addition to the 3-harmonic filter. The analysis of CoRoT's photometric series did not reveal the need to use the fourth harmonic. However, the analysis of the spectrographic parameters associated with the activity of the star (mainly $\log R_{\mathrm{hk}}$ ) showed the importance of highorder harmonics even beyond the fourth. In addition, the proximity of the fourth harmonic to an alias of the period of CoRoT-7b makes its introduction necessary.

The coincidence between the period of the fourth rotation harmonic (5.91 d) and an alias of the orbital period of CoRoT$7 \mathrm{~b}(5.925 \mathrm{~d})$ is a major problem in this study. We have to stress first that, in series of astronomical observations done at the same hour angle, it is impossible to solve any aliasing problem. In such a case, a linear relationship appears between the two components. We may force the value of one component and compensate with the value of the other. There is no mathematical tool able to solve this problem. In the case of CoRoT-7b, given the slight difference between the two frequencies (5.91 and 5.925) and the complexity of the signal, the only way to get one solution is to break the uniformity of the time intervals in the sample by means of observations done at very different hour angles. Unfortunately, this is not easy with observations from only one observatory. Differences of a few hours with respect to the mean are the maximum that could have been obtained in this case. The set of observations selected for this study has 52 observations done in 27 nights, including ten nights with three observations in a four hour interval and five nights with two observations. This is the only hope that we have for solving the beat of the two frequencies before new observations are done. They were indeed separated using a 4-harmonic, high-pass filter, and the convergence of the self-consistent iteration routes to the same result (see Fig. 3) is an indication that, in the adopted algorithm, they may be considered as independent.

One additional comment with respect to the beat comes from the analysis of Fig. 6 (top). That figure shows the difference between the estimated activity in the two cases: with three and four harmonics. It is an evaluation of the contribution of the fourth harmonic to the estimated activity of the star. The surprise, in this case, is that the apparent period of this contribution is not $5.91 \mathrm{~d}$, but only $5.25 \mathrm{~d}$. Assuming that the rotation period is the same as observed by CoRoT, this difference would mean that we are not dealing with one frozen periodic signal, but with a signal that is just nearly periodic: as the evolution of a periodic process whose period and phase are continuously changing. The other possibility is that the period is not the same as the published one. We note that 5.25 is one fourth of the main period found in the free Fourier analysis of the radial velocities, 21.15 days, and that the frequency of the highest peak in the Fourier spectrum of the data, in Fig. 7 is near $0.5 \mathrm{~d}^{-1}$ instead of $0.042 \mathrm{~d}^{-1}$, the inverse of $23.64 \mathrm{~d}^{6}$. Regardless of the reason for this difference, it certainly contributed to the 4-harmonic filter succeeding to get one solution with the two components separated, while classical leastsquares solution and a covariance analysis using the filtered RV obtained with the published rotation was unable to separate the orbital period of CoRoT-7b from the alias of fourth harmonic. As a check, we did some runs of our codes using this lower period. The results are masses near the values obtained using the actual values of the rotation period, so they confirm those results and show that the filtering algorithm is robust with respect to variations in the rotation period used in the filter, at least inasmuch as it is close to the adopted ones.

The quality of the results may be assessed from the residuals $(\mathrm{O}-\mathrm{C})$ and from the fitting of the observations to the model shown in Fig. 11. The obtained $(\mathrm{O}-\mathrm{C})$ correspond to a w.r.m.s. of $1.9 \mathrm{~m} \mathrm{~s}^{-1}$. Two comments may be added here: (1) the error bars in Fig. 11 are larger than those of the RV measurements. They result from the addition of the variances in the RV measurements to that of the activity given by the used model. The large errors in some dates in the middle of the interval correspond to dates having only one observation per night, leading to larger statistical errors in estimating the activity in these dates; (2) these results naturally absorb RV jumps like those that led Pont et al. (2010) to state that HARPS systematic errors may be huge in some cases. One of the $20 \mathrm{~m} \mathrm{~s}^{-1}$ jumps reported by them, which occurred between the dates BJD $2454868 / 69$ (BJD - $2454820=48 / 49)$, is in the studied interval. However, in our results, it is mainly the consequence of a strong increase in the star RV due to the reaction to the combined motion of the two planets on those dates. When the increase due to the activity (about $3 \mathrm{~m} \mathrm{~s}^{-1}$ ) is added to it, one may verify that the part of the unexplained RV increase is still large, but just one third of the increase seen in the RV measurements.

\footnotetext{
6 The variation from 23.64 to 21,1 days in the activity period is consistent with differential rotation differences expected in a solar-type star (see Thomas \& Weiss 2008). However, the time span of the observations considered here is not long enough to allow us to give full credit to this result and the question should be reconsidered when new data become available.
} 


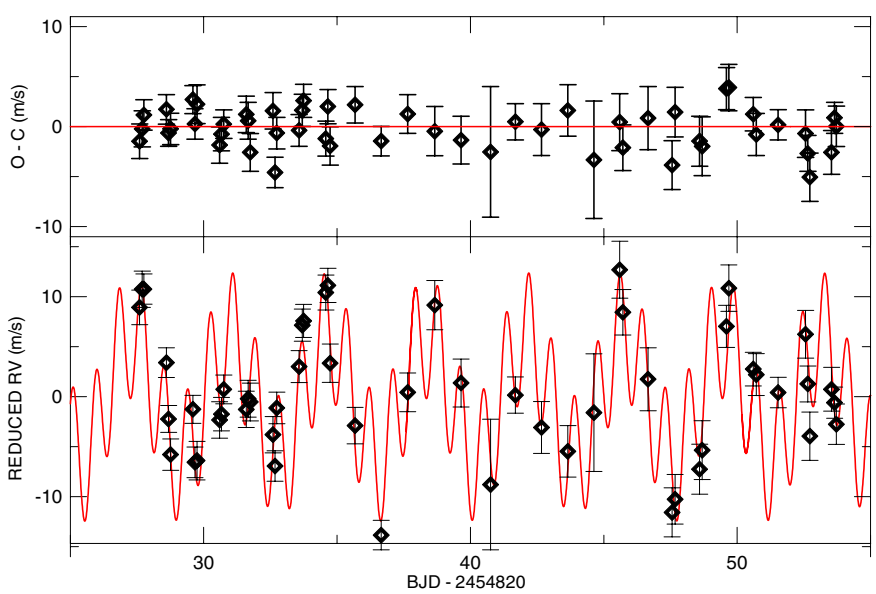

Fig. 11. Bottom: filtered radial velocities with the 4-harmonic high-pass filter and 14-day coherence time (dots) and RV curve corresponding to the planets solution obtained with the same parameters. Top: differences between the filtered RV and the RV curve.

Table 2. Masses and elements (Epoch BJD 2454 820.0).

\begin{tabular}{ccc}
\hline \hline & CoRot-7b & CoRot-7c \\
\hline $\mathrm{K}\left(\mathrm{m} \mathrm{s}^{-1}\right)$ & $5.7 \pm 0.8$ & $6.0 \pm 0.6$ \\
Mass (Earth Mass) & $8 \pm 1.2$ & $13.6 \pm 1.4$ \\
Longitude at Epoch (deg) $_{\text {Period (d) (fixed) }}^{\dagger}$ & $207 \pm 4$ & $225 \pm 3$ \\
Period (d) (estimated) & 0.8535855 (see text) & 3.698 \\
T0 (BJD) (estimated) & $2454847.893 \pm 0.009$ & \\
Eccentricity (fixed) & 0 & 0 \\
\hline
\end{tabular}

Notes. ${ }^{(\dagger)}$ The mass of CoRoT $7 \mathrm{c}$ is minimal $(\times \sin i)$.

An alternative approach suggested by Hatzes et al. (2010), which has the advantage of offering no explicit hypotheses on the activity (except its slow variation), has also been improved and combined with a biased Monte Carlo approach. The resulting mass of CoRoT-7b lies close to results obtained using the 3-harmonic filter. The resulting mass of CoRoT-7c is not determined well because the period of CoRoT-7c is not short enough to allow it to be fully separated from the star activity.

\section{Conclusions}

The final results were obtained by combining the results of the 3- and 4-harmonic filtering and of the multiple-observations-pernight alternative approach. They are given in Table 2 . The results from filtering were considered when the two codes used were convergent and led to similar values. Otherwise they were not considered in the final estimation of the results. In particular we mention that the largest and least coherence times (22 and 8, respectively) were not considered. The other criterion was the quality of the filtering as indicated by the comparison of the Fourier transforms before and after the filtering (Fig. 7).

We recall that, because of the short timespan of the observations used, we decide not to determine the periods and used those given in the discovery papers (Léger et al. 2009, and QBM). With the period determined from the transits and the date of the first transit of CoRoT-7b observed by CoRoT, we obtained 196.4 degrees for the expected longitude at the epoch BJD 2454820.0 . The small offset with respect to the result shown in Table 2 corresponds to a slight correction in the period, which would then be $0.85354 \pm 0.00002$ days.
We also stress that the errors given in Table 2 intend to define the interval in which the two masses are expected to be. They are not the result of any popular statistical formulas. Those formulas are generally valid in conditions that are much more restrictive than the actual ones, and often the results are much smaller than the actual errors. An additional difficulty in the results for CoRoT-7c comes from that it is difficult to disentangle the mass of this planet from the higher rotation harmonics (mainly the 6th). In the case of the beats between the period of CoRoT-7b and the 4th harmonic, it was possible to disentangle them by making multiple observations in many of the nights of the latest observation periods. In the case of CoRoT$7 \mathrm{c}$, the beats are not related to aliasing, and the problems they raise cannot be solved by the strategy of making multiple observations per night or by mathematical tools allowing low- and high-frequency components to be separated. To solve this kind of entanglement we should have observations spanning a longer time interval. We did some calculations using all observations available in the interval BJD 2554825-884, by increasing the time span from 27 to 60 nights. This means including discontinuities in the observation set, which makes the analysis more difficult and, in some sense, less certain. In these runs, the mass obtained for CoRoT-7c is below 13 Earth masses. Adding this to the evidence from the Fourier analysis of the residuals used in the runs labeled as $4 \mathrm{H}$, that the 6th rotation harmonic may contribute with $0.5 \mathrm{~m} \mathrm{~s}^{-1}$ to $K_{2}$, we may guess that values between 13 and 14 Earth masses are the more probable ones. One may wish that the improvement in techniques such as the one recently proposed by Pont et al. (2011) applied to simultaneous photometric and spectrographic observation over long time spans give a final answer to this question in the future. However, with the existing observations, we are confident that the above estimates are the best ones we can obtain and that all consistent estimations fall in the ranges given above or, at least, in its immediate neighborhood.

The longitudes at BJD $=2454820$ were obtained in the same way as the masses, by comparing the solutions from various models (see Fig. 12). The individual statistical errors are 5 and 12 degrees for planets CoRoT 7b and 7c, respectively. The other two approaches did not contribute to the given results because of too large errors.

One last point is that the result found here for CoRoT $7 \mathrm{~b}$ means that the planet has a bulk density of $11 \pm 3.5 \mathrm{~g} / \mathrm{cm}^{3}$. This is much more than the $6.6 \mathrm{~g} / \mathrm{cm}^{3}$ resulting from the mass given in QBM and means not only that CoRoT 7b is rocky, but also that the contribution from the iron core must be higher (between 50 and 65 percent in mass; see Fortney et al. 2007; Seager et al. 2007 ) and that the density at its center may be close to $25 \mathrm{~g} / \mathrm{cm}^{3}$ (see Seager et al. 2007). These values are higher than those corresponding to other known rocky planets, but all consistent determinations of the mass of CoRoT-7b lead to bulk densities of at least $9 \mathrm{~g} / \mathrm{cm}^{3}$ showing that the high-density of CoRoT-7b is a constraint to be taken into account in modeling the planet.

The comparison of the various methods used in this paper allows us to say that the high-pass filter used by QBM, embedded in a self-consistent algorithm, is the best one we can devise to disentangle long- and short-period terms in a given series of unevenly spaced observations. Its superiority over Fourier analyses with fixed frequencies comes from the use of a running window allowing the method to treat a signal that is not periodic or has a period different from the one used in the filter. The procedure proposed by Hatzes et al. (2010) of using only nights with multiple observations and including an additional $V_{0}$ for each date is an important complement. It suffers, however, 
S. Ferraz-Mello et al.: Planetary mass determination of super-Earths orbiting active stars

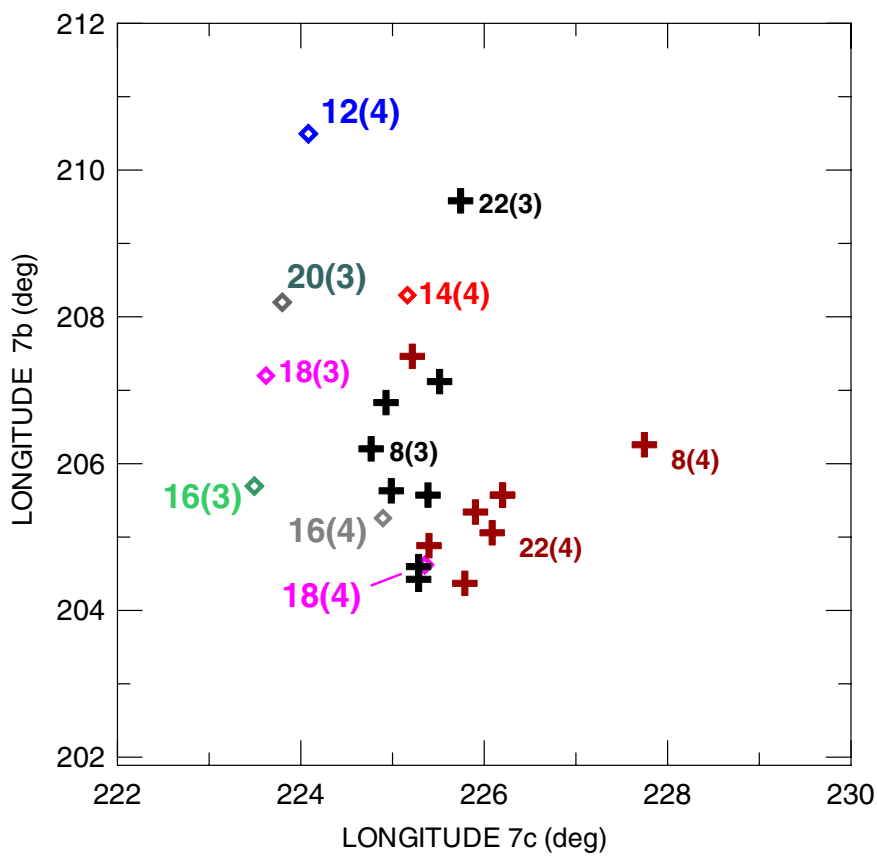

Fig. 12. Longitudes at the epoch $B J D=2454820$ resulting from several runs of the iterative filtering. The labels indicate the number of harmonics in the high-pass filter and the coherence time in days. For the results obtained using the genetic algorithm + simplex code (crosses), only some results are labeled to avoid overlaps.

from a shortcoming due to the great number of unknowns that it involves. As a consequence, the number of degrees of freedom is small, and the resulting confidence intervals are too large. In the current case, it only allowed us to determine the mass of CoRoT 7b. This was enough because this was the ultimate goal of our work, but the impossibility of getting reasonable confidence intervals for the mass of CoRoT 7c needs to be mentioned. The planning of new observations should take this into account and have as many multiple-night observations as possible.
Acknowledgements. S.F.M. and C.B. acknowledge the fellowships of the Isaac Newton Institute for Mathematical Sciences, University of Cambridge (UK) where they have developed the tools used to investigate this problem. M.T.S. acknowledge FAPESP. The continuous support of CNPq (grants 302783/20075 and 485447/2007-0) and the CAPES-SECYT Brazil-Argentina joint science program (grant 131-07) are also acknowledged. In the course of this investigation, several members of the CoRoT Exoplanets Science Team have supplied us with relevant information on the RV data and their analysis. We want particularly thank A. Hatzes and D. Queloz for their help. We are pleased to acknowledge the excellent reviewer's report, raising many questions and contributing enormously to improving the paper.

\section{References}

Batalha, N., Borucki, W. J., Bryson, S. T., et al. 2011, ApJ, 729, 27

Boisse, I., Bouchy, F., Hébrad, G., et al. 2011, A\&A, 528, A4

Bruntt, H., Deleuil, M., Fridlund, M., et al. 2010, A\&A, 519, A51

Charbonneau, D., Berta, Z., Irwin, J., et al. 2009, Nature, 462, 891

Cumming, A., Marcy, G., \& Butler, P. 1999, ApJ, 526, 890

Ferraz-Mello, S. 1981, AJ, 86, 619

Ferraz-Mello, S., Michtchenko, T. A., \& Beaugé, C. 2005, ApJ, 621, 473

Ferraz-Mello, S., Rodríguez, A., \& Hussmann, H. 2008, Celest. Mech. Dyn. Astron., 101, 171

Fortney, J. J., Marley, M. S., \& Barnes, J. W. 2007, ApJ, 659, 1661

Hatzes, A., Dvorak, R., Wuchterl, G., et al. 2010, A\&A, 520, A93

Lanza, A., Bonomo, A., Moutou, C., et al. 2010, A\&A, 520, A53

Léger, A., Rouan, D., Schneider, J., et al. 2009, A\&A, 506, 287

Linnik, Y. V. 1961, Méthode des moindres carrés, Éléments de la théorie du traitement des observations, Dunod, Paris

Mardling, R. A. 2007, MNRAS, 382, 1768

Mardling, R. A. 2010, MNRAS, 407, 1048

Mignard, F. 1979, Moon and Planets, 20, 301

Pont, F., Aigrain, S., \& Zucker, S. 2011, MNRAS, 411, 1953

Press, W. H., Flannery, B. P., Teukolsky, S. A., \& Vetterling, W. T. 1986, Numerical Recipes (Cambridge, UK: CUP)

Queloz, D., Bouchy, F., Moutou, C., et al. 2009, A\&A, 506, 303

Rodríguez, A. 2010, Ph.D. dissertation, Univ. of São Paulo

Rodríguez, A., Ferraz-Mello, S., Michtchenko, T. A., Beaugé, C., \& Miloni, O. 2011, MNRAS [arXiv: 1104.0964]

Seager, S., Kuchner, M., Hier-Majumder, C. A., \& Militzer, B. 2007, ApJ, 669, 1279

Thomas, J. H., \& Weiss, N. O. 2008, Sunspots and Starspots (Cambridge, UK: CUP) 